\title{
Multivariate analogue of slant Toeplitz operators
}

\author{
Gopal Datt*1 (D), Shesh Kumar Pandey² (D) \\ ${ }^{1}$ Department of Mathematics, PGDAV College, University of Delhi, Delhi-110065, India \\ ${ }^{2}$ Department of Mathematics, University of Delhi, Delhi-11000\%, India
}

\begin{abstract}
This paper discusses several structural and fundamental properties of the $k^{\text {th }}$-order slant Toeplitz operators on the Lebesgue space of the $n$ - torus $\mathbb{T}^{n}$, for integers $k \geq 2$ and $n \geq 1$. We obtain certain equivalent conditions for the commutativity and essential commutativity of these operators. In the last section, we deal with the spectrum of a $k^{\text {th }}$-order slant Toeplitz operator on $L^{2}\left(\mathbb{T}^{n}\right)$ and investigate the conditions for such an operator to be an isometry, hyponormal or normal.
\end{abstract}

Mathematics Subject Classification (2020). 47B35, 46E30, 46E35

Keywords. commutativity, Lebesgue space, slant Toeplitz operators, n-dimensional torus

\section{Introduction}

The class of Toeplitz operators possesses extensively vast literature, which contains immense informations and implications. The algebraic and $C^{*}$ - algebraic view points of Toeplitz operators have resulted into profound and adequate theory. Toeplitz operators were introduced in the commencement of 20 th century by O. Toeplitz. The theory of Toeplitz operators is related with different areas like $C^{*}$-algebra, function theory and certain other fields of operator theory. A matrix $A=\left(a_{i, j}\right)_{i, j \geq 0}$ is called a Toeplitz matrix if $a_{i, j}=a_{i+1, j+1}$. Toeplitz operator on $H^{2}(\mathbb{T})$, the Hardy-Hilbert space, is defined to be the compression of a multiplication operator on $L^{2}(\mathbb{T})$ to the space $H^{2}(\mathbb{T})$, where $\mathbb{T}$ is the unit circle in the complex plane $\mathbb{C}$. Over the years, several researchers have given different generalizations of Toeplitz operators. Toeplitz and Hankel operators [10] exhibit crucial roles in the study of each other, together with their significant features.

Ho $[7,8]$ introduced the notion of a slant Toeplitz operator on $L^{2}(\mathbb{T})$, which is closely related with the multiplication operator. He derived several structural properties of these operators. The study of $k^{t h}$-order slant Toeplitz operators on $L^{2}(\mathbb{T})$ and its compression to $H^{2}(\mathbb{T})$ is initiated by Arora and Batra [1]. For adequate literature on these operators and their applications to wavelets and dynamical systems, one is referred to $[6,7,10]$ and the references therein. The theory of Toeplitz operators to the Hardy space of the bi-disk was developed by many mathematicians (see $[2,4,5,9]$ ).

\footnotetext{
*Corresponding Author.

Email addresses: gopal.d.sati@gmail.com (G. Datt), sheshkumar.1992@gmail.com (S.K. Pandey)

Received: 23.12.2019; Accepted: 13.10.2020
} 
Let $\mathbb{D}^{n}$ be the open unit polydisc in $\mathbb{C}^{n}$ and $\mathbb{T}^{n}$, the distinguished boundary of $\mathbb{D}^{n}$, denotes the $n$ - torus. Although, the function theory on the polydisc (see [11]) is significantly different from the one on the unit disc, the available theory of multiple Fourier series on the $n$ - torus enables one to discuss function spaces (e.g. $\left.L^{2}\left(\mathbb{T}^{n}\right), L^{\infty}\left(\mathbb{T}^{n}\right)\right)$ as well as slant Toeplitz operators in the higher dimensional setting. In the whole paper, the space of all Lebesgue integrable functions on $\mathbb{T}^{n}$ and the collection of all Lebesgue square integrable functions on $\mathbb{T}^{n}$ are respectively denoted by $L^{1}\left(\mathbb{T}^{n}\right)$ and $L^{2}\left(\mathbb{T}^{n}\right)$. The class of all essentially bounded measurable functions on $\mathbb{T}^{n}$ is expressed by $L^{\infty}\left(\mathbb{T}^{n}\right)$. Any two functions in these spaces are equal in the sense of equality almost everywhere. The Fourier coefficients of $f \in L^{1}\left(\mathbb{T}^{n}\right)$ are given by

$$
f_{m_{1}, m_{2}, \ldots, m_{n}}=\frac{1}{(2 \pi)^{n}} \underbrace{\int_{0}^{2 \pi} \int_{0}^{2 \pi} \cdots \int_{0}^{2 \pi}}_{n \text {-times }} f\left(e^{i \theta_{1}}, \ldots, e^{i \theta_{n}}\right) e^{-i\left(m_{1} \theta_{1}+\ldots+m_{n} \theta_{n}\right)} d \theta_{1} d \theta_{2} \ldots d \theta_{n},
$$

for $m_{i} \in \mathbb{Z}, 1 \leq i \leq n$. If the Fourier coefficient $f_{m_{1}, m_{2}, \ldots, m_{n}}=0$ for each $\left(m_{1}, m_{2}, \ldots, m_{n}\right) \in$ $\mathbb{Z}^{n}$, then $f \equiv 0$ (see [12, Theorem 1.7 (Chapter VII)]). The multiple Fourier series can be seen or treated as Fourier transformation of functions in $L^{1}\left(\mathbb{T}^{n}\right)$. Therefore, by the multiple Fourier series [12] on $\mathbb{T}^{n}$, the spaces $L^{2}\left(\mathbb{T}^{n}\right)$ and $H^{2}\left(\mathbb{T}^{n}\right)$ can be written as

$$
\begin{gathered}
L^{2}\left(\mathbb{T}^{n}\right)=\left\{f: f\left(z_{1}, z_{2}, \ldots, z_{n}\right)=\sum_{\left(m_{1}, m_{2}, \cdots, m_{n}\right) \in \mathbb{Z}^{n}} f_{m_{1}, m_{2}, \cdots, m_{n}} z_{1}^{m_{1}} z_{2}^{m_{2}} \cdots z_{n}^{m_{n}},\right. \\
\left.\sum_{\left(m_{1}, m_{2}, \cdots, m_{n}\right) \in \mathbb{Z}^{n}}\left|f_{m_{1}, m_{2}, \cdots, m_{n}}\right|^{2}<\infty\right\},
\end{gathered}
$$

and

$$
\begin{gathered}
H^{2}\left(\mathbb{T}^{n}\right)=\left\{f: f\left(z_{1}, z_{2}, \ldots, z_{n}\right)=\sum_{\left(m_{1}, m_{2}, \cdots, m_{n}\right) \in \mathbb{Z}_{+}^{n}} f_{m_{1}, m_{2}, \cdots, m_{n}} z_{1}^{m_{1}} z_{2}^{m_{2}} \cdots z_{n}^{m_{n}},\right. \\
\left.\sum_{\left(m_{1}, m_{2}, \cdots, m_{n}\right) \in \mathbb{Z}_{+}^{n}}\left|f_{m_{1}, m_{2}, \cdots, m_{n}}\right|^{2}<\infty\right\},
\end{gathered}
$$

where $\mathbb{Z}$ and $\mathbb{Z}_{+}$are respectively the set of all integers and the set of all non-negative integers. Clearly, the space $L^{2}\left(\mathbb{T}^{n}\right)$ is a Hilbert space with the norm given by the inner product

$$
\langle f, g\rangle=\frac{1}{(2 \pi)^{n}} \underbrace{\int_{0}^{2 \pi} \int_{0}^{2 \pi} \cdots \int_{0}^{2 \pi}}_{n \text {-times }} f\left(e^{i \theta_{1}}, e^{i \theta_{2}}, \cdots, e^{i \theta_{n}}\right) \overline{g\left(e^{i \theta_{1}}, e^{i \theta_{2}}, \cdots, e^{i \theta_{n}}\right)} d \theta_{1} d \theta_{2} \ldots d \theta_{n} .
$$

The class $\left\{e_{m_{1}, m_{2}, \cdots, m_{n}}:\left(m_{1}, m_{2}, \ldots, m_{n}\right) \in \mathbb{Z}_{+}^{n}\right\}$, where $e_{m_{1}, \cdots, m_{n}}\left(z_{1}, z_{2} \cdots, z_{n}\right)=z_{1}^{m_{1}}$ $z_{2}^{m_{2}} \ldots z_{n}^{m_{n}}$, deduces an orthonormal basis of $H^{2}\left(\mathbb{T}^{n}\right)$. The space $H^{2}\left(\mathbb{T}^{n}\right)$ can be shown as a closed subspace of $L^{2}\left(\mathbb{T}^{n}\right)$ such that for $f \in H^{2}\left(\mathbb{T}^{n}\right)$,

$$
\left\langle f, e_{m_{1}, m_{2}, \ldots, m_{n}}\right\rangle=0 \text { if } m_{j}<0 \text { for at least one } j, 1 \leq j \leq n .
$$

Many mathematicians use multi-index notations namely $\boldsymbol{d} \boldsymbol{\theta}, \boldsymbol{z}^{\boldsymbol{m}}$ and $\boldsymbol{m}$ for $d \theta_{1} d \theta_{2} \ldots d \theta_{n}$, $z_{1}^{m_{1}} z_{2}^{m_{2}} \ldots z_{n}^{m_{n}}$ and $\left(m_{1}, m_{2}, \ldots, m_{n}\right)$ respectively. However, we prefer the latter notations to have better visualization of functions in multi-variable cases. From now on, an analytic function $f$ in $L^{2}\left(\mathbb{T}^{n}\right)$ is a function, whose Fourier coefficients $f_{m_{1}, m_{2}, \ldots, m_{n}}=0$, whenever $m_{j}<0$ for at least one $j, 1 \leq j \leq n$. Note that here the notion of an analytic function is not the same as in the case of complex plane. By a co-analytic function in $L^{2}\left(\mathbb{T}^{n}\right)$ we mean a function whose complex conjugate is analytic in the above sense. Also, the 
symbol $\mathbb{Z}_{k}$ stands for the set $\{0,1,2, \ldots, k-1\}$ for $k \geq 2$. Motivated by the work of Ho $[7,8]$, we extended the notion of slant Toeplitz operators to the space $L^{2}\left(\mathbb{T}^{n}\right)$ in [3]. In this paper, we discuss various properties of $k^{t h}$-order slant Toeplitz operators and obtain results describing spectra and spectral radii of these operators.

\section{Commuting operators}

This section is mainly devoted to the study of symbols inducing commuting $k^{\text {th }}$-order slant Toeplitz operators. We begin with the following known notions.

Definition 2.1 ([8]). A slant Toeplitz operator $A_{\phi}$, induced by symbol $\phi \in L^{\infty}(\mathbb{T})$, on the Lebesgue space $L^{2}(\mathbb{T})$ is given by $A_{\phi}=E M_{\phi}$, where $M_{\phi}$ is the multiplication operator on $L^{2}(\mathbb{T})$ with symbol $\phi$, and the operator $E$ is the linear operator on $L^{2}(\mathbb{T})$ defined by

$$
E\left(z^{m}\right)= \begin{cases}z^{m / 2} & \text { if } m \text { is an even integer } \\ 0, & \text { otherwise. }\end{cases}
$$

Definition 2.2 ([3]). For $\phi \in L^{\infty}\left(\mathbb{T}^{n}\right)$ and an integer $k \geq 2$, a $k^{t h}$-order slant Toeplitz operator $A_{\phi, k, n}$ with symbol $\phi$, on the Lebesgue space $L^{2}\left(\mathbb{T}^{n}\right)$, is defined to be $A_{\phi, k, n}=$ $E_{k, n} M_{\phi}$, where $M_{\phi}$ is the multiplication operator on $L^{2}\left(\mathbb{T}^{n}\right)$ induced by $\phi$, and the operator $E_{k, n}$ is the linear operator on $L^{2}\left(\mathbb{T}^{n}\right)$ given by

$$
E_{k, n}\left(z_{1}^{i_{1}} z_{2}^{i_{2}} \ldots z_{n}^{i_{n}}\right)= \begin{cases}z_{1}^{\frac{i_{1}}{k}} z_{2}^{\frac{i_{2}}{k}} \ldots z_{n}^{\frac{i_{n}}{k}} & \text { if each } i_{j} \text { is a multiple of } \mathrm{k}, \text { for } 1 \leq j \leq n \\ 0, & \text { otherwise }\end{cases}
$$

In fact, the operator $E_{k, n}$ is a bounded linear operator on $L^{2}\left(\mathbb{T}^{n}\right)$ with norm $\left\|E_{k, n}\right\|=1$. The $k^{t h}$-order slant Toeplitz operator $A_{\phi, k, n}$ is also a bounded linear operator on $L^{2}\left(\mathbb{T}^{n}\right)$ such that $\left\|A_{\phi, k, n}\right\| \leq\|\phi\|_{\infty}$. The operator $A_{1, k, n}$, induced by $\phi \equiv 1$, is nothing but $E_{k, n}$. In particular, for $k=2$, the operator $A_{\phi, 2, n}$ is known as slant Toeplitz operator on $L^{2}\left(\mathbb{T}^{n}\right)$. In [3], it is proved that a bounded operator $A$ on $L^{2}\left(\mathbb{T}^{n}\right)$ is a $k^{t h}$-order slant Toeplitz operator if and only if $A$ satisfies the following conditions:

$$
M_{z_{j}} A=A M_{z_{j}}, \quad 1 \leq j \leq n .
$$

Now, we are in the position to analyze the commutativity of slant Toeplitz operators. Initially, we start with a result related to $E_{k, n}$. For the basic properties of $E_{k, n}$, we refer [3].

Proposition 2.3. For any two integers $k$ and $m(\geq 2), E_{k, n} E_{m, n}=E_{k m, n}$ on the space $L^{2}\left(\mathbb{T}^{n}\right)$.

Proof. In order to furnish the proof of the result, for any $n$-tuple $\left(i_{1}, i_{2}, \ldots, i_{n}\right) \in \mathbb{Z}^{n}$, we observe that

$$
\begin{aligned}
E_{k, n} E_{m, n}\left(z_{1}^{i_{1}} z_{2}^{i_{2}} \ldots z_{n}^{i_{n}}\right) & = \begin{cases}z_{1}^{\frac{i_{1}}{k m}} z_{2}^{\frac{i_{2}}{k m}} \ldots z_{n}^{\frac{i_{n}}{k m}} & \text { if each } i_{j} \text { is a multiple of } k m, \text { for } \\
0, & \begin{array}{c}
1 \leq j \leq n \\
\text { otherwise. }
\end{array} \\
& =E_{k m, n}\left(z_{1}^{i_{1}} z_{2}^{i_{2}} \ldots z_{n}^{i_{n}}\right) .\end{cases}
\end{aligned}
$$

The above observation gives that $E_{k, n} E_{m, n}=E_{k m, n}$ for all integers $k, m \geq 2$.

In view of preceding result, one can point out the following conclusion without any extra effort.

Corollary 2.4. The operators $E_{k, n}$ and $E_{m, n}$ commute for all integers $k, m \geq 2$. 
The next theorem is an attempt towards commutativity of $k^{\text {th }}$-order slant Toeplitz operators induced by different symbols and which are of different orders. More precisely, the following theorem helps to find out the condition on the inducing function so that $k^{\text {th }}$-order slant Toeplitz operators commute.

Lemma 2.5. Let $k, m \geq 2$ be any two integers and $\phi, \psi$ be functions from the space $L^{\infty}\left(\mathbb{T}^{n}\right)$. Then $A_{\phi, k, n} A_{\psi, m, n}=A_{\Psi, k m, n}$, where $\Psi$ is the function in $L^{\infty}\left(\mathbb{T}^{n}\right)$ given by $\Psi\left(z_{1}, z_{2}, \ldots, z_{n}\right)=\phi\left(z_{1}^{m}, z_{2}^{m}, \ldots, z_{n}^{m}\right) \psi\left(z_{1}, z_{2}, \ldots, z_{n}\right)$.

Proof. Let $\phi, \psi \in L^{\infty}\left(\mathbb{T}^{n}\right)$ and $k, m \in \mathbb{Z}$ such that $k, m \geq 2$. Then, by [3, Proposition 3.2], the operator $A_{\phi, k, n} A_{\psi, m, n}$ can be written as

$$
\begin{aligned}
A_{\phi, k, n} A_{\psi, m, n} & =E_{k, n} M_{\phi} E_{m, n} M_{\psi} \\
& =E_{k, n} E_{m, n} M_{\phi\left(z_{1}^{m}, z_{2}^{m}, \ldots, z_{n}^{m}\right)} M_{\psi} \\
& =E_{k m, n} M_{\phi\left(z_{1}^{m}, z_{2}^{m}, \ldots, z_{n}^{m}\right) \psi} \\
& =A_{\phi\left(z_{1}^{m}, z_{2}^{m}, \ldots, z_{n}^{m}\right) \psi, k m, n},
\end{aligned}
$$

which is in the desired form. This completes the proof.

The assertion obtained in the following result helps significantly to arrive at the conclusion that the commutativity and essential commutativity for $k^{\text {th }}$-order slant Toeplitz operators are equivalent. To deduce the following output, we utilize the fact that $A_{\phi, k, n}$ is compact if and only if $\phi=0$ if and only if $A_{\phi, k, n}=0$, which is proved in [3].

Theorem 2.6. Let $\phi, \psi \in L^{\infty}\left(\mathbb{T}^{n}\right)$ and $k, m$ be two integers such that $k, m \geq 2$. Then, the following statements are equivalent

(1) The operator $A_{\phi, k, n} A_{\psi, m, n}$ is a compact operator.

(2) $A_{\phi, k, n} A_{\psi, m, n}=0$.

(3) $\phi\left(z_{1}^{m}, z_{2}^{m}, \ldots, z_{n}^{m}\right) \psi=0$.

Proof. In view of relation (2.1), the operator $A_{\phi, k, n} A_{\psi, m, n}$ can be expressed as

$$
A_{\phi, k, n} A_{\psi, m, n}=E_{k m, n} M_{\phi\left(z_{1}^{m}, z_{2}^{m}, \ldots, z_{n}^{m}\right) \psi} .
$$

This expression gives that the operator $A_{\phi, k, n} A_{\psi, m, n}$ is a $k m$-th order slant Toeplitz operator, induced by $\phi\left(z_{1}^{m}, z_{2}^{m}, \ldots, z_{n}^{m}\right) \psi$. With the help of [3, Proposition 2.8 and Theorem $2.13]$, one can easily conclude the desired equivalent statements.

An immediate output can be derived from the latter is that the product of two $k^{\text {th }}$-order slant Toeplitz operators induced by $\phi, \psi \in L^{\infty}\left(\mathbb{T}^{n}\right)$ is a compact operator if and only if $\phi\left(z_{1}^{k}, z_{2}^{k}, \ldots, z_{n}^{k}\right) \psi$ is the zero function. Using the preceding result appropriately, it is easy to obtain the following

Corollary 2.7. Let $\phi, \psi$ be two functions from the space $L^{\infty}\left(\mathbb{T}^{n}\right)$. Then, the following are equivalent:

(1) The operators $A_{\phi, k, n}$ and $A_{\psi, m, n}$ essentially commute.

(2) The operators $A_{\phi, k, n}$ and $A_{\psi, m, n}$ commute.

(3) $\phi\left(z_{1}^{m}, z_{2}^{m}, \ldots, z_{n}^{m}\right) \psi-\phi \psi\left(z_{1}^{k}, z_{2}^{k}, \ldots, z_{n}^{k}\right)=0$.

Proof. In order to prove the result, consider the following expression

$$
\begin{aligned}
A_{\phi, k, n} A_{\psi, m, n}-A_{\psi, m, n} A_{\phi, k, n} & =E_{k m, n} M_{\phi\left(z_{1}^{m}, z_{2}^{m}, \ldots, z_{n}^{m}\right) \psi}-E_{k m, n} M_{\phi \psi\left(z_{1}^{k}, z_{2}^{k}, \ldots, z_{n}^{k}\right)} \\
& =E_{k m, n} M_{\phi\left(z_{1}^{m}, z_{2}^{m}, \ldots, z_{n}^{m}\right) \psi-\phi \psi\left(z_{1}^{k}, z_{2}^{k}, \ldots, z_{n}^{k}\right)} .
\end{aligned}
$$

The result follows by the use of Theorem 2.6 and essential commutativity and commutativity of operators.

In particular, for $k=m$, Corollary 2.7 lead to the following conclusions about the $k^{t h}$-order slant Toeplitz operators $A_{\phi, k, n}$ and $A_{\psi, k, n}$. 
Corollary 2.8. The following statements are equivalent for $\phi, \psi \in L^{\infty}\left(\mathbb{T}^{n}\right)$ :

(1) $A_{\phi, k, n} A_{\psi, k, n}=A_{\psi, k, n} A_{\phi, k, n}$.

(2) $A_{\phi, k, n}$ and $A_{\psi, k, n}$ essentially commute.

(3) $\phi\left(z_{1}^{k}, z_{2}^{k}, \ldots, z_{n}^{k}\right) \psi-\psi\left(z_{1}^{k}, z_{2}^{k}, \ldots, z_{n}^{k}\right) \phi=0$.

If $\phi \in L^{\infty}\left(\mathbb{T}^{n}\right)$, given by

$$
\phi\left(z_{1}, z_{2}, \ldots, z_{n}\right)=\sum_{\left(m_{1}, m_{2}, \ldots, m_{n}\right) \in \mathbb{Z}^{n}} \phi_{m_{1}, m_{2}, \ldots, m_{n}} z_{1}^{m_{1}} z_{2}^{m_{2}} \ldots z_{n}^{m_{n}}
$$

satisfies $\phi\left(z_{1}^{k}, z_{2}^{k}, \ldots, z_{n}^{k}\right)=\phi$ then for each $\left(i_{1}, i_{2}, \ldots, i_{n}\right) \in \mathbb{Z}^{n}$, it can be seen that

$$
\begin{aligned}
0=\left\langle\phi\left(z_{1}^{k}, z_{2}^{k}, \ldots, z_{n}^{k}\right)-\phi\left(z_{1}, z_{2}, \ldots, z_{n}\right), z_{1}^{i_{1}} z_{2}^{i_{2}} \ldots z_{n}^{i_{n}}\right\rangle \\
=\left\{\begin{array}{lc}
\phi_{\frac{i_{1}}{k}, \frac{i_{2}}{k}, \ldots, \frac{i_{n}}{k}}-\phi_{i_{1}, i_{2}, \ldots, i_{n}}, & \text { if each } i_{j} \text { is a multiple of } k, 1 \leq j \leq n \\
-\phi_{i_{1}, i_{2}, \ldots, i_{n}}, & \text { otherwise. }
\end{array}\right.
\end{aligned}
$$

The more significant form of the above expression is the following

$$
\phi_{i_{1}, i_{2}, \ldots, i_{n}}= \begin{cases}\phi_{\frac{i_{1}}{k}, \frac{i_{2}}{k}, \ldots, \frac{i_{n}}{k},} & \text { if each } i_{j} \text { is a multiple of } k, \text { for } 1 \leq j \leq n \\ 0, & \text { otherwise. }\end{cases}
$$

If each $i_{j}$ is a multiple of $k$ for $1 \leq j \leq n$. Then, by using equation (2.2), it can be obtained that $\phi_{i_{1}, i_{2}, \ldots, i_{n}}=\phi_{m_{1}, m_{2}, \ldots, m_{n}}$ such that at least one $m_{j}, 1 \leq j \leq n$, is not divisible by $k$. Therefore, in view of (2.2) again, we get that $\phi_{i_{1}, i_{2}, \ldots, i_{n}}=0$ for all nonzero $n$-tuple $\left(i_{1}, i_{2}, \ldots, i_{n}\right) \in \mathbb{Z}^{n}$, which gives that $\phi=\phi_{0,0, \ldots, 0}$ (constant function).

We are aware with the fact that $M_{\phi} E_{k, n}=A_{\phi\left(z_{1}^{k}, z_{2}^{k}, \ldots, z_{n}^{k}\right), k, n}$, which leads us to a conclusion that $E_{k, n}$ and $M_{\phi}$ commute if and only if $\phi$ is a constant. In the next result, we intend to establish a generalized version of the above mentioned conclusion.

Theorem 2.9. The operators $M_{\phi}$ and $A_{\psi, k, n}$ commute if and only if the expression $\phi\left(z_{1}^{k}, z_{2}^{k}, \ldots, z_{n}^{k}\right) \psi=\phi \psi$ is satisfied for $\phi, \psi \in L^{\infty}\left(\mathbb{T}^{n}\right)$. Further, if $\psi$ is invertible, then the following statements are equivalent:

(1) $M_{\phi} A_{\psi, k, n}=A_{\psi, k, n} M_{\phi}$

(2) $\phi$ is a constant function.

Proof. To prove the desired equivalent statements, consider the operators $A_{\psi, k, n} M_{\phi}$ and $M_{\phi} A_{\psi, k, n}$, which can be respectively written as

$$
A_{\psi, k, n} M_{\phi}=E_{k, n} M_{\psi \phi}=A_{\psi \phi, k, n} \quad \text { and } \quad M_{\phi} A_{\psi, k, n}=A_{\phi\left(z_{1}^{k}, z_{2}^{k}, \ldots, z_{n}^{k}\right) \psi, k, n} .
$$

The preceding expressions and the injectivity of the mapping $\left(\phi \longmapsto A_{\phi, k, n}\right)$, which is shown in [3], are enough to declare that $M_{\phi} A_{\psi, k, n}=A_{\psi, k, n} M_{\phi}$ if and only if $\phi\left(z_{1}^{k}, z_{2}^{k}, \ldots, z_{n}^{k}\right)$ $\psi=\phi \psi$.

Further, we assume that $\psi$ is an invertible function of $L^{\infty}\left(\mathbb{T}^{n}\right)$, then by the above observation, it is evident that $M_{\phi} A_{\psi, k, n}=A_{\psi, k, n} M_{\phi}$ is equivalent to $\phi\left(z_{1}^{k}, z_{2}^{k}, \ldots, z_{n}^{k}\right)=\phi$. This completes the proof.

Being $E_{k, n}$ a $k^{t h}$-order slant Toeplitz operator, the above theorem provides an immediate inference that the operator $E_{k, n}$ and the multiplication operator $M_{\phi}$, induced by $\phi \in$ $L^{\infty}\left(\mathbb{T}^{n}\right)$, commute if and only if $\phi$ is a constant.

\section{Properties}

This section highlights certain structural properties, which are associated to $k^{\text {th }}$-order slant Toeplitz operator on $L^{2}\left(\mathbb{T}^{n}\right)$. It also deals with $C^{*}$-algebraic aspects linked to the $k^{\text {th }}$-order slant Toeplitz operators. Ultimately, the section includes significant observations made for spectral properties of $k^{t h}$-order slant Toeplitz operators. 
The following theorem reveals the connection between weighted composition operators and $k^{t h}$-order slant Toeplitz operators. The weighted composition $C_{\phi, H}$ is a bounded operator on $L^{2}\left(\mathbb{T}^{n}\right)$ given by $C_{\phi, H}(f)=\bar{\phi} \cdot(f \circ H)$, where $H$ is a mapping from $\mathbb{T}^{n}$ to itself such that $H\left(z_{1}, z_{2}, \ldots, z_{n}\right)=\left(z_{1}^{k}, z_{2}^{k}, \ldots, z_{n}^{k}\right)$.

Theorem 3.1. Let $\phi$ be an element of the space $L^{\infty}\left(\mathbb{T}^{n}\right)$. Then, $A_{\phi, k, n}^{*}$, the adjoint of $A_{\phi, k, n}$, is a weighted composition operator $C_{\phi, H}$, where $H$ is defined as above.

Proof. Suppose that $\phi \in L^{\infty}\left(\mathbb{T}^{n}\right)$ is of the form

$$
\phi\left(z_{1}, z_{2}, \ldots, z_{n}\right)=\sum_{\left(m_{1}, m_{2}, \ldots, m_{n}\right) \in \mathbb{Z}^{n}} \phi_{m_{1}, m_{2}, \ldots, m_{n}} z_{1}^{m_{1}} z_{2}^{m_{2}} \ldots z_{n}^{m_{n}} .
$$

As we know that the action of the adjoint $A_{\phi, k, n}^{*}$ of $k^{\text {th }}$-order slant Toeplitz operator on basis elements of $L^{2}\left(\mathbb{T}^{n}\right)$ is given by

$$
\begin{aligned}
A_{\phi, k, n}^{*} e_{i_{1}, \ldots, i_{n}}\left(z_{1}, z_{2}, \ldots, z_{n}\right) & =\sum_{\left(m_{1}, m_{2} \ldots, m_{n}\right) \in \mathbb{Z}^{n}} \bar{\phi}_{k i_{1}-m_{1}, k i_{2}-m_{2}, \ldots, k i_{n}-m_{n}} z_{1}^{m_{1}} z_{2}^{m_{2}} \ldots z_{n}^{m_{n}} \\
& =\sum_{\left(m_{1}, m_{2} \ldots, m_{n}\right) \in \mathbb{Z}^{n}} \bar{\phi}_{m_{1}, m_{2}, \ldots, m_{n}} z_{1}^{k i_{1}-m_{1}} z_{2}^{k i_{2}-m_{2}} \ldots z_{n}^{k i_{n}-m_{n}} \\
& =\left[\bar{\phi}\left(z_{1}, z_{2}, \ldots, z_{n}\right)\right] \cdot\left[e_{i_{1}, i_{2}, \ldots, i_{n}} \circ H\left(z_{1}, z_{n}, \ldots, z_{n}\right)\right],
\end{aligned}
$$

for each $\left(i_{1}, i_{2}, \ldots, i_{n}\right) \in \mathbb{Z}^{n}$. By the use of linearity of $A_{\phi, k, n}^{*}$, we get that $A_{\phi, k, n}^{*}(f)=$ $\bar{\phi} \cdot(f \circ H)=C_{\phi, H}(f)$. This completes the proof.

We begin with an observation that if $\phi \in L^{\infty}\left(\mathbb{T}^{n}\right)$ then so is $E_{k, n}(\phi)$. To show the desired conclusion, we utilize the frequently used fact that $E_{k, n} M_{\phi} E_{k, n}^{*}=M_{E_{k, n}(\phi)}$, which is already seen in [2, Lemma 3.12]. It gives that

$$
E_{k, n} A_{\phi, k, n}^{*}=E_{k, n} M_{\bar{\phi}} E_{k, n}^{*}=M_{E_{k, n}(\bar{\phi})} .
$$

Since the operator $E_{k, n} A_{\phi, k, n}^{*}$ is bounded, hence $M_{E_{k, n}(\bar{\phi})}$ is bounded. This implies that $E_{k, n}(\bar{\phi}) \in L^{\infty}\left(\mathbb{T}^{n}\right)$.

By a positive function on $\mathbb{T}^{n}$, we mean a function $\phi$ on $\mathbb{T}^{n}$ such that $\phi\left(z_{1}, z_{2}, \ldots, z_{n}\right) \geq 0$ almost everywhere and in this case we write $\phi \geq 0$ almost everywhere. As a consequence of the preceding observation, we have the following.

Theorem 3.2. If $\phi$ is an element of $L^{\infty}\left(\mathbb{T}^{n}\right)$ such that $\phi \geq 0$ almost everywhere. Then, $E_{k, n}(\phi) \geq 0$ almost everywhere on $\mathbb{T}^{n}$.

Proof. Let $\phi$ be a positive element of the space $L^{\infty}\left(\mathbb{T}^{n}\right)$. For the accomplishment of the proof, consider the operator $A_{\sqrt{\phi}, k, n} A_{\sqrt{\phi}, k, n}^{*}$, which can be expressed as

$$
A_{\sqrt{\phi}, k, n} A_{\sqrt{\phi}, k, n}^{*}=E_{k, n} M_{|\sqrt{\phi}|^{2}} E_{k, n}^{*}=M_{E_{k, n}(\phi)} .
$$

Since the operator $A_{\sqrt{\phi}, k, n} A_{\sqrt{\phi}, k, n}^{*}$ is positive, therefore $M_{E_{k, n}(\phi)}$ is also positive. We know that the multiplication operator is positive if and only if the inducing function is positive. Hence, in view of the above observation, $E_{k, n}(\phi)$ is a positive element of $L^{\infty}\left(\mathbb{T}^{n}\right)$. This completes the proof.

The above theorem can be rephrased as the operator $E_{k, n}$ sends a positive element of $L^{\infty}\left(\mathbb{T}^{n}\right)$ into a positive element of the same space, which provides a trivial consequence in the following form.

Corollary 3.3. Let $\phi, \psi$ be two functions of the space $L^{\infty}\left(\mathbb{T}^{n}\right)$ such that $\phi \geq \psi \geq 0$ almost everywhere. Then, $E_{k, n}(\phi) \geq E_{k, n}(\psi)$ almost everywhere on $\mathbb{T}^{n}$. 
In the paper [3], it is already shown that $\left\|A_{\phi, k, n}^{m}\right\|=\left\|\Phi_{m}\right\|_{\infty}^{\frac{1}{2}}$ and $r\left(A_{\phi, k, n}\right)=\lim _{m \rightarrow \infty}\left\|\Phi_{m}\right\|_{\infty}^{\frac{1}{2 m}}$, where $m \in \mathbb{N}, r\left(A_{\phi, k, n}\right)$ denotes the spectral radius of $A_{\phi, k, n}$ and $\Phi_{m}$ can be expressed as

$$
\Phi_{m}=\underbrace{E_{k, n}\left(| \phi | ^ { 2 } E _ { k , n } \left(| \phi | ^ { 2 } E _ { k , n } \left(\ldots E_{k, n}\right.\right.\right.}_{m \text {-times }}\left(|\phi|^{2}\right) \ldots)))=A_{|\phi|^{2}, k, n}^{m}(1) .
$$

In particular, the norm of $A_{\phi, k, n}$ is given as $\left\|A_{\phi, k, n}\right\|=\left\|E_{k, n}\left(|\phi|^{2}\right)\right\|_{\infty}^{\frac{1}{2}}$. This observation brings out the following.

Corollary 3.4. Let $\phi$ be an inner function. Then, the spectral radius of $A_{\phi, k, n}$ induced by $\phi$ is 1 .

Now, we would like to derive a relation between the spectral radii of operators $A_{\phi, k, n}$ and $A_{\psi, k, n}$ for $\phi, \psi \in L^{\infty}\left(\mathbb{T}^{n}\right)$ such that $|\phi| \geq|\psi| \geq 0$ almost everywhere (in short a.e.).

Theorem 3.5. If $\phi, \psi$ are two elements of $L^{\infty}\left(\mathbb{T}^{n}\right)$ with the condition that $|\phi| \geq|\psi| \geq 0$ almost everywhere on $\mathbb{T}^{n}$. Then, $\left\|A_{\phi, k, n}^{p}\right\| \geq\left\|A_{\psi, k, n}^{p}\right\|$ and $r\left(A_{\phi, k, n}\right) \geq r\left(A_{\psi, k, n}\right)$, where $p \in \mathbb{N}$.

Proof. Suppose that $\phi, \psi$ are functions in $L^{\infty}\left(\mathbb{T}^{n}\right)$ such that $|\phi| \geq|\psi| \geq 0$ a.e. on $\mathbb{T}^{n}$, which gives that $|\phi|^{2} \geq|\psi|^{2} \geq 0$ a.e. on $\mathbb{T}^{n}$. By the use of Theorem 3.2, one can have

$$
E_{k, n}\left(|\phi|^{2}\right) \geq E_{k, n}\left(|\psi|^{2}\right) \quad \text { a.e. on } \mathbb{T}^{n} \text {. }
$$

Similarly, it is easy to see that

$$
E_{k, n}\left[|\phi|^{2} E_{k, n}\left(|\phi|^{2}\right)\right] \geq E_{k, n}\left[|\psi|^{2} E_{k, n}\left(|\psi|^{2}\right)\right] \quad \text { a.e. on } \mathbb{T}^{n} .
$$

Equivalently, we get that $\Phi_{2} \geq \Psi_{2}$ a.e. Again, in a similar way, one can show that $\Phi_{p} \geq \Psi_{p}$ a.e. for $p \in \mathbb{N}$, where $\Phi_{p}$ and $\Psi_{p}$ are given by (3.1). In view of [2, Lemma 3.12 and Theorem 3.13], the preceding relation yields the desired result.

By a trigonometric polynomial on $\mathbb{T}^{n}$, we mean a function $\phi$ on $\mathbb{T}^{n}$ with Fourier series of the form

$$
\phi\left(z_{1}, z_{2}, \ldots, z_{n}\right)=\sum_{\substack{\left(m_{1}, m_{2}, \ldots, m_{n}\right) \in \mathbb{Z}^{n} \\-M_{i} \leq m_{i} \leq M_{i}, 1 \leq i \leq n}} \phi_{m_{1}, m_{2}, \ldots, m_{n}} z_{1}^{m_{1}} z_{2}^{m_{2}} \ldots z_{n}^{m_{n}},
$$

where $M_{i}$ 's are nonnegative integers. Equivalently, without loss of generality, trigonometric polynomial $\phi$ on $\mathbb{T}^{n}$ can be defined as

$$
\phi\left(z_{1}, z_{2}, \ldots, z_{n}\right)=\sum_{\substack{\left(m_{1}, m_{2}, \ldots, m_{n}\right) \in \mathbb{Z}^{n} \\-M \leq m_{i} \leq M, 1 \leq i \leq n}} \phi_{m_{1}, m_{2}, \ldots, m_{n}} z_{1}^{m_{1}} z_{2}^{m_{2}} \ldots z_{n}^{m_{n}},
$$

where $M$ is a nonnegative integer.

Next, we look for invariant subspaces of the $k^{t h}$-order slant Toeplitz operator with symbol $\phi$ being a trigonometric polynomial.

Lemma 3.6. Let $\phi$ be a trigonometric polynomial given by (3.2). Then, we have the following

(1) The closed linear subspace $H_{\phi}$ of $L^{2}\left(\mathbb{T}^{n}\right)$ spanned by $\left\{z_{1}^{i_{1}} z_{2}^{i_{2}} \ldots z_{n}^{i_{n}}:\left|i_{j}\right| \leq(k-\right.$ 1) $M$ for $1 \leq j \leq n\}$ is invariant under $A_{\phi, k, n}$.

(2) The function $\Phi_{p}=A_{|\phi|^{2}, k, n}^{p}$ (1), given by (3.1), is contained in the finite dimensional subspace $H_{|\phi|^{2}}$ generated by $\left\{z_{1}^{i_{1}} z_{2}^{i_{2}} \ldots z_{n}^{i_{n}}:\left|i_{j}\right| \leq 2(k-1) M\right.$ for $\left.1 \leq j \leq n\right\}$. 
Proof. For the accomplishment of the proof, operate $A_{z_{1}^{m_{1}} z_{2}^{m_{2}} \ldots z_{n}^{m_{n}}, k, n}$ on the elements of $H_{\phi}$, which gives that

$A_{z_{1}^{m_{1}} z_{2}^{m_{2}} \ldots z_{n}^{m_{n}}, k, n}\left(z_{1}^{i_{1}} z_{2}^{i_{2}} \ldots z_{n}^{i_{n}}\right)= \begin{cases}z_{1}^{\frac{m_{1}+i_{1}}{k}} z_{2}^{\frac{m_{2}+i_{2}}{k}} \ldots z_{n}^{\frac{m_{n}+i_{n}}{k}}, & \text { if } i_{j} \text { 's and } m_{j} \text { 's are multiple } \\ 0, & \text { of } k \text { for } 1 \leq j \leq n \\ 0, & \text { otherwise. }\end{cases}$

Since $\left|\frac{m_{j}+i_{j}}{k}\right| \leq M$ for each $j$ such that $1 \leq j \leq n$. Therefore, we obtain that $H_{\phi}$ is invariant under $A_{z_{1}^{m_{1}} z_{2}^{m_{2}} \ldots z_{n}^{m_{n}}, k, n}$ and hence under $A_{\phi, k, n}$.

The second part is an immediate consequence of part (1).

The next theorem reveals the relationship between the double commutant of the class of $k^{t h}$-order slant Toeplitz operators and collection of all bounded operators on $L^{2}\left(\mathbb{T}^{n}\right)$.

Lemma 3.7. Let $\mathcal{S}$ be the $C^{*}$-algebra generated by all $k$-th order slant Toeplitz operators on $L^{2}\left(\mathbb{T}^{n}\right)$. Then, the double commutant of $\mathcal{S}$ is the collection $\mathfrak{B}\left(L^{2}\left(\mathbb{T}^{n}\right)\right)$ of all bounded operators on $L^{2}\left(\mathbb{T}^{n}\right)$.

Proof. Let $\mathcal{M}$ denotes the $C^{*}$-algebra generated by all multiplication or Laurent operators on $L^{2}\left(\mathbb{T}^{n}\right)$. Then, by [2, Lemma 2.11(ii)], one can observe that $\mathcal{M} \subseteq \mathcal{S}$. Therefore, $\mathcal{S}^{\prime} \subseteq \mathcal{M}^{\prime}=\mathcal{M}$, where $\mathcal{M}^{\prime}$ and $\mathcal{S}^{\prime}$ represent the commutants of the $C^{*}$-algebras $\mathcal{M}$ and $\mathcal{S}$ respectively. Let $T$ be any element in $\mathcal{S}^{\prime}$. Then, by the preceding inclusion, $T$ is a Laurent operator $M_{\phi}$ for some inducing function $\phi \in L^{\infty}\left(\mathbb{T}^{n}\right)$. Since, $E_{k, n}$ is a $k^{t h}$-order slant Toeplitz operator with symbol $\phi \equiv 1$, one can point out that $T E_{k, n}=E_{k, n} T$ i.e. $M_{\phi} E_{k, n}=E_{k, n} M_{\phi}$. Consequently, the Theorem 2.9 derives that $\phi$ is a constant and hence $T$ is a constant multiple of $I$, the identity operator. This implies that $\mathcal{S}^{\prime}=\mathcal{J}$, where $\mathcal{J}$ is the $C^{*}$-algebra generated by the identity operator. Hence, the required result follows.

Apparently, the preceding lemma and Von-Neumann double commutant theorem yield the following.

Theorem 3.8. The $C^{*}$-algebra (unital) $\mathcal{S}$ generated by the collection of all $k$-th order slant Toeplitz operators is $\mathfrak{B}\left(L^{2}\left(\mathbb{T}^{n}\right)\right)$.

In the following results, we discuss hyponormality, normality, co-isometric, partial isometric and isometric behaviors of our operators.

Theorem 3.9. A necessary and sufficient condition for a $k$-th order slant Toeplitz operator $A_{\phi, k, n}$ to be a partial isometry is that $\phi=\phi \cdot E_{k, n}^{*} E_{k, n}\left(|\phi|^{2}\right)$. In particular, if $\phi$ is invertible then $A_{\phi, k, n}$ is a partial isometry if and only if $E_{k, n}^{*} E_{k, n}\left(|\phi|^{2}\right)=1$.

Proof. We know that an operator $T$ is a partial isometry if and only if $T=T T^{*} T$, which gives that $A_{\phi, k, n}$ is a partial isometry if and only if $A_{\phi, k, n}=A_{\phi, k, n} A_{\phi, k, n}^{*} A_{\phi, k, n}$. Now, consider the following expression

$$
\begin{aligned}
A_{\phi, k, n} & =A_{\phi, k, n} A_{\phi, k, n}^{*} A_{\phi, k, n} \\
& =E_{k, n} M_{\phi} M_{\bar{\phi}} E_{k, n}^{*} E_{k, n} M_{\phi} \\
& =M_{E_{k, n}\left(|\phi|^{2}\right)} E_{k, n} M_{\phi} \\
& =A_{\left[\phi \cdot E_{k, n}\left(|\phi|^{2}\right)\left(z_{1}^{k}, \ldots, z_{n}^{k}\right)\right], k, n}=A_{\left[\phi \cdot E_{k, n}^{*} E_{k, n}\left(|\phi|^{2}\right)\right], k, n},
\end{aligned}
$$

which yields that the operator $A_{\phi, k, n}$ is a partial isometry if and only if it satisfies $A_{\phi, k, n}=$ $A_{\left[\phi \cdot E_{k, n}^{*} E_{k, n}\left(|\phi|^{2}\right)\right], k, n}$. The injectivity of the mapping $\phi \longmapsto A_{\phi, k, n}$, helps to provide the required result. The particular case of the theorem is straightforward.

An illustration in the confirmation of the above theorem is the following. 
Example 3.10. The operator $A_{\phi, k, n}$ is a partial isometry for $\phi\left(z_{1}, z_{2}, \ldots, z_{n}\right)=\frac{1}{2} z_{1} z_{2}$ $\ldots z_{n}+\frac{\sqrt{3}}{2} z_{1}^{k} z_{2}^{k} \ldots z_{n}^{k}$. One can easily verify that $\phi \in L^{\infty}\left(\mathbb{T}^{n}\right)$ and that $|\phi|^{2}=1+$ $\frac{\sqrt{3}}{4}\left(z_{1}^{k-1} z_{2}^{k-1} \ldots z_{n}^{k-1}\right)+\frac{\sqrt{3}}{4}\left(z_{1}^{-k+1} z_{2}^{-k+1} \ldots z_{n}^{-k+1}\right)$, which yields that $E_{k, n}|\phi|^{2}=1$ and hence $\phi E_{k, n}^{*} E_{k, n}|\phi|^{2}=\phi$. Therefore, the desired conclusion follows in view of the above theorem.

The next result investigates inducing functions $\phi$, which give isometric $k^{t h}$-order slant Toeplitz operators $A_{\phi, k, n}$.

Theorem 3.11. A $k^{\text {th }}$-order slant Toeplitz operator on $L^{2}\left(\mathbb{T}^{n}\right)$ cannot be isometric.

Proof. If possible, assume that a $k^{t h}$-order slant Toeplitz operator $A_{\phi, k, n}$ induced by $\phi$ is an isometry, where $\phi$ is of the form

$$
\phi\left(z_{1}, z_{2}, \ldots, z_{n}\right)=\sum_{\left(m_{1}, m_{2}, \ldots, m_{n}\right) \in \mathbb{Z}^{n}} \phi_{m_{1}, m_{2}, \ldots, m_{n}} z_{1}^{m_{1}} z_{2}^{m_{2}} \ldots z_{n}^{m_{n}} .
$$

Then, for $i_{j} \in \mathbb{Z}_{k}, 1 \leq j \leq n$, we get that $\left\|A_{\phi, k, n} z_{1}^{i_{1}} z_{2}^{i_{2}} \ldots z_{n}^{i_{n}}\right\|=\left\|z_{1}^{i_{1}} z_{2}^{i_{2}} \ldots z_{n}^{i_{n}}\right\|=1$, which can be rewritten as

$$
\sum_{\left(m_{1}, m_{2}, \ldots, m_{n}\right) \in \mathbb{Z}^{n}}\left|\phi_{k m_{1}-i_{1}, k m_{2}-i_{2}, \ldots, k m_{n}-i_{n}}\right|^{2}=1 .
$$

Also, from the equation (3.3), the norm of $\phi$ in $L^{2}\left(\mathbb{T}^{n}\right)$ becomes

$$
\begin{aligned}
\|\phi\|^{2} & =\sum_{\left(m_{1}, m_{2}, \ldots, m_{n}\right) \in \mathbb{Z}^{n}}\left|\phi_{m_{1}, m_{2}, \ldots, m_{n}}\right|^{2} \\
& =\sum_{i_{j}=0,1 \leq j \leq n}^{k-1} \sum_{\left(m_{1}, m_{2}, \ldots, m_{n}\right) \in \mathbb{Z}^{n}}\left|\phi_{k m_{1}-i_{1}, k m_{2}-i_{2}, \ldots, k m_{n}-i_{n}}\right|^{2}=k^{n} .
\end{aligned}
$$

Hence, we obtain that $\left\|E_{k, n}\left(|\phi|^{2}\right)\right\|=\left\|A_{\phi, k, n}(\bar{\phi})\right\|=\|\phi\|=k^{\frac{n}{2}}$. But, we know that $\left\|A_{\phi, k, n}\right\|=\left\|E_{k, n}\left(|\phi|^{2}\right)\right\|_{\infty}^{\frac{1}{2}}$ and $A_{\phi, k, n}$ is an isometry. This implies that $\left\|E_{k, n}\left(|\phi|^{2}\right)\right\|_{\infty}=1$. Since $k \geq 2$ and $n \geq 1$, so the above observation helps to conclude that

$$
\left\|E_{k, n}\left(|\phi|^{2}\right)\right\|_{\infty}<\left\|E_{k, n}\left(|\phi|^{2}\right)\right\|_{2}
$$

which is a contradiction. Hence the conclusion of the theorem follows.

In the following example, we construct an isometric operator with the help of $k^{\text {th }}$-order slant Toeplitz operator.

Example 3.12. Let $\alpha, \beta \in \mathbb{C}$ and $\phi\left(z_{1}, z_{2}, \ldots, z_{n}\right)=\alpha\left(z_{1} z_{2} \ldots z_{n}\right)+\beta$. Then, one can observe that $A_{\phi, k, n} A_{\phi, k, n}^{*}=M_{E_{k, n}\left(|\phi|^{2}\right)}$ and

$$
E_{k, n}\left(|\phi|^{2}\right)=E_{k, n}\left(|\alpha|^{2}+|\beta|^{2}+\alpha \bar{\beta} z_{1}, z_{2}, \ldots, z_{n}+\bar{\alpha} \beta \overline{\left(z_{1}, z_{2}, \ldots, z_{n}\right)}\right)=|\alpha|^{2}+|\beta|^{2} .
$$

Therefore, the operator $\frac{1}{\sqrt{|\alpha|^{2}+|\beta|^{2}}} A_{\phi, k, n}^{*}$ is an isometry.

Theorem 3.13. The zero operator is the only hyponormal $k^{\text {th }}$-order slant Toeplitz operatoron $L^{2}\left(\mathbb{T}^{n}\right)$.

Proof. Let $\phi \in L^{\infty}\left(\mathbb{T}^{n}\right)$ be given by

$$
\phi\left(z_{1}, z_{2}, \ldots, z_{n}\right)=\sum_{\left(m_{1}, m_{2}, \ldots, m_{n}\right) \in \mathbb{Z}^{n}} \phi_{m_{1}, m_{2}, \ldots, m_{n}} z_{1}^{m_{1}} z_{2}^{m_{2}} \ldots z_{n}^{m_{n}} .
$$

Suppose that $A_{\phi, k, n}$ is a hyponormal operator induced by $\phi$. Then, we have

$$
\left\|A_{\phi, k, n} f\right\| \geq\left\|A_{\phi, k, n}^{*} f\right\| \quad \text { for all } f \in L^{2}\left(\mathbb{T}^{n}\right) .
$$


In particular, for the function $f \equiv 1$, the relation (3.4) gives that

$$
\sum_{\left(m_{1}, m_{2}, \ldots, m_{n}\right) \in \mathbb{Z}^{n}}\left|\phi_{k m_{1}, k m_{2}, \ldots, k m_{n}}\right|^{2} \geq \sum_{\left(m_{1}, m_{2}, \ldots, m_{n}\right) \in \mathbb{Z}^{n}}\left|\phi_{-m_{1},-m_{2}, \ldots,-m_{n}}\right|^{2},
$$

which yields that $\phi_{k m_{1}-i_{1}, k m_{2}-i_{2}, \ldots, k m_{n}-i_{n}}=0$ for all $i_{j} \in \mathbb{Z}_{k}, 1 \leq j \leq n$ but $\left(i_{1}, i_{2}, \ldots, i_{n}\right)$ $\neq(0,0, \ldots, 0)$. Also, for $f=z_{1} z_{2} \ldots z_{n}$, the relation (3.4) reduces to

$$
\sum_{\left(m_{1}, m_{2}, \ldots, m_{n}\right) \in \mathbb{Z}^{n}}\left|\phi_{k m_{1}-1, k m_{2}-1, \ldots, k m_{n}-1}\right|^{2} \geq \sum_{\left(m_{1}, m_{2}, \ldots, m_{n}\right) \in \mathbb{Z}^{n}}\left|\phi_{k-m_{1}, k-m_{2}, \ldots, k-m_{n}}\right|^{2},
$$

In view of the above observation, inequality (3.6) helps to conclude that $\phi_{k m_{1}, k m_{2}, \ldots}$ $\ldots, k m_{n}=0$. Thus, we obtain that $\phi=0$ and hence $A_{\phi, k, n}=0$.

Since an isometry is always a hyponormal operator, Theorem 3.11 turns out to be a particular case of Theorem 3.13. Moreover, one can also draw the following conclusion from Theorem 3.13.

Corollary 3.14. The only normal $k^{\text {th }}$-order slant Toeplitz operatoron $L^{2}\left(\mathbb{T}^{n}\right)$ is the zero operator.

Now, we intend to establish a condition on the inducing function so that $A_{\phi, k, n}$ is a co-isometry.

Theorem 3.15. A $k^{\text {th }}$-order slant Toeplitz operator $A_{\phi, k, n}$ on $L^{2}\left(\mathbb{T}^{n}\right)$ is a co-isometry if and only if $E_{k, n}\left(|\phi|^{2}\right)=1$.

Proof. We know that an operator $T$ is a co-isometry if and only if $T T^{*}=I$. Now, consider the expression $A_{\phi, k, n} A_{\phi, k, n}^{*}=E_{k, n} M_{\phi} M_{\bar{\phi}} E_{k, n}^{*}=M_{E_{k, n}\left(|\phi|^{2}\right)}$, which provides that $A_{\phi, k, n}$ on $L^{2}\left(\mathbb{T}^{n}\right)$ is a co-isometry if and only if $E_{k, n}\left(|\phi|^{2}\right)=1$. This completes the proof.

Now, the following conclusion can be immediately drawn.

Corollary 3.16. The $k^{\text {th }}$-order slant Toeplitz operator $A_{\phi, k, n}$ is always a co-isometry for a unimodular inducing function $\phi \in L^{\infty}\left(\mathbb{T}^{n}\right)$.

The following examples verify the existence of co-isometric $k^{\text {th }}$-order slant Toeplitz operators.

Example 3.17. Consider the $k^{t h}$-order slant Toeplitz operator $A_{\phi, k, n}$ induced by $\phi$, where $\phi\left(z_{1}, z_{2}, \ldots, z_{n}\right)=\frac{1}{\sqrt{1+|\alpha|^{2}}}\left(z_{1} z_{2} \ldots z_{n}+\alpha\right), \alpha \in \mathbb{C}$. Clearly, $\phi \in L^{\infty}\left(\mathbb{T}^{n}\right)$ and $E_{k, n}\left(|\phi|^{2}\right)=$ 1. Therefore, in view of Corollary 3.16 , one can conclude that $A_{\phi, k, n}$ is a co-isometry.

Example 3.18. The operator $A_{\phi, k, n}$, where $\phi$ is as in Example 3.10, is a co-isometric $k^{\text {th }}$-order slant Toeplitz operator.

Theorem 3.19. A necessary and sufficient condition for a $k^{\text {th }}$-order slant Toeplitz operator $A_{\phi, k, n}$ on $L^{2}\left(\mathbb{T}^{n}\right)$ to be a co-isometry is that

$$
\sum_{r_{j}=0,1 \leq j \leq n}^{k-1}\left|\phi\left(\frac{\theta_{1}+2 r_{1} \pi}{k}, \frac{\theta_{2}+2 r_{2} \pi}{k}, \ldots, \frac{\theta_{n}+2 r_{n} \pi}{k}\right)\right|^{2}=k^{n} \text { on } \mathbb{T}^{n} \text { a.e.. }
$$

Proof. In order to obtain the required condition, we use the fact that an operator $T$ is a co-isometry if and only if $T^{*}$ preserves the norm. For each $f \in L^{2}\left(\mathbb{T}^{n}\right)$, computation gives 
the following

$$
\begin{aligned}
\left\|A_{\phi, k, n}^{*}(f)\right\|^{2}= & \left\|\bar{\phi} \cdot E_{k, n}^{*}(f)\right\|^{2} \\
= & \frac{1}{(2 \pi)^{n}} \underbrace{\int_{0}^{2 \pi} \int_{0}^{2 \pi} \cdots \int_{0}^{2 \pi}}_{n-\text { times }}\left|\phi\left(\theta_{1}, \ldots, \theta_{n}\right)\right|^{2}\left|f\left(k \theta_{1}, \ldots, k \theta_{n}\right)\right|^{2} d \theta_{1} \ldots d \theta_{n} \\
= & \frac{1}{(2 k \pi)^{n}} \underbrace{\int_{0}^{2 k \pi} \ldots \int_{0}^{2 k \pi}}_{n-\text { times }}\left|\phi\left(\frac{\theta_{1}}{k}, \ldots, \frac{\theta_{n}}{k}\right)\right|^{2}\left|f\left(\theta_{1}, \ldots, \theta_{n}\right)\right|^{2} d \theta_{1} \ldots d \theta_{n} \\
= & \frac{1}{(2 k \pi)^{n}} \underbrace{\int_{0}^{2 \pi} \int_{0}^{2 \pi} \ldots \int_{0}^{2 \pi}}_{n-\text { times }} \sum_{r_{j}=0,1 \leq j \leq n}^{k-1}\left|\phi\left(\frac{\theta_{1}+2 r_{1} \pi}{k}, \ldots, \frac{\theta_{n}+2 r_{n} \pi}{k}\right)\right|^{2} \\
& \times\left|f\left(\theta_{1}, \theta_{2}, \ldots, \theta_{n}\right)\right|^{2} d \theta_{1} d \theta_{2} \ldots d \theta_{n} \\
= & \left\|M_{\psi}(f)\right\|^{2},
\end{aligned}
$$

where $\psi\left(\theta_{1}, \theta_{2}, \ldots, \theta_{n}\right)=\sqrt{\frac{1}{k^{n}} \sum_{r_{j}=0,1 \leq j \leq n}^{k-1}\left|\phi\left(\frac{\theta_{1}+2 r_{1} \pi}{k}, \frac{\theta_{2}+2 r_{2} \pi}{k}, \ldots, \frac{\theta_{n}+2 r_{n} \pi}{k}\right)\right|^{2}}$. By the above computation, the result follows without any extra effort because of the fact that $\left\|M_{\psi}(f)\right\|=$ $\|f\|$ if and only if $\psi$ is unimodular.

The preceding theorem along with Theorem 3.15 can be drafted in the following form.

Theorem 3.20. For a $k^{\text {th }}$-order slant Toeplitz operator $A_{\phi, k, n}$ on $L^{2}\left(\mathbb{T}^{n}\right)$, the following are equivalent:

(1) $A_{\phi, k, n}$ is a co-isometry.

(2) $E_{k, n}\left(|\phi|^{2}\right)=1$.

(3) $\sum_{r_{j}=0,1 \leq j \leq n}^{k-1}\left|\phi\left(\omega^{r_{1}} z_{1}, \omega^{r_{2}} z_{2}, \ldots, \omega^{r_{n}} z_{n}\right)\right|^{2}=k^{n}$ on $\mathbb{T}^{n}$ almost everywhere, where $\omega$ is a $k^{\text {th }}$-root of unity.

In subsequent results, we focus on the study of the spectrum of $k^{\text {th }}$-order slant Toeplitz operator. In particular, it will be shown that a closed disc is contained in the spectrum of $A_{\phi, k, n}$.

Lemma 3.21. Let $\phi \in L^{\infty}\left(\mathbb{T}^{n}\right)$ be invertible. Then, the kernel of $A_{\phi, k, n}$ is the closed span of $S$ given by

$$
S=\left\{\left(z_{1}^{k m_{1}-p_{1}} z_{2}^{k m_{2}-p_{2}} \ldots z_{n}^{k m_{n}-p_{n}}\right) \phi^{-1}: m_{i} \in \mathbb{Z},(0,0, \ldots, 0) \neq\left(p_{1}, p_{2}, \ldots, p_{n}\right) \in \mathbb{Z}_{k}^{n}\right\} .
$$

Proof. Initially, we claim that the range $R\left(A_{\phi, k, n}^{*}\right)$ is closed, which follows immediately because of the fact that $M_{\bar{\phi}}$ is invertible and $E_{k, n}^{*}$ is an isometry. We know that $R\left(A_{\phi, k, n}^{*}\right)^{\perp}=$ $\operatorname{ker}\left(A_{\phi, k, n}\right)$, provided that $R\left(A_{\phi, k, n}^{*}\right)$ is closed. Therefore, we make an attempt to prove that $R\left(A_{\phi, k, n}^{*}\right)^{\perp}=S$. One can observe that the range of $A_{\phi, k, n}^{*}$ is the closed span of $S_{0}=$ $\left\{\bar{\phi} \cdot\left(z_{1}^{k m_{1}} z_{2}^{k m_{2}} \ldots z_{n}^{k m_{n}}\right): m_{i} \in \mathbb{Z}, 1 \leq i \leq n\right\}$. Now, for $\left(i_{1}, i_{2}, \ldots, i_{n}\right),\left(j_{1}, j_{2}, \ldots, j_{n}\right) \in \mathbb{Z}^{n}$ and $p_{m} \in \mathbb{Z}_{k}$ but not all $p_{m}=0$, we have

$$
\begin{aligned}
\left\langle\bar{\phi} \cdot\left(z_{1}^{k i_{1}} z_{2}^{k i_{2}} \ldots z_{n}^{k i_{n}}\right), \phi^{-1} \cdot\left(z_{1}^{k j_{1}-p_{1}} z_{2}^{k j_{2}-p_{2}} \ldots z_{n}^{k j_{n}-p_{n}}\right)\right\rangle & \\
& =\left\langle\left(z_{1}^{k i_{1}} z_{2}^{k i_{2}} \ldots z_{n}^{k i_{n}}\right),\left(z_{1}^{k j_{1}-p_{1}} z_{2}^{k j_{2}-p_{2}} \ldots z_{n}^{k j_{n}-p_{n}}\right)\right\rangle=0 .
\end{aligned}
$$

It gives that $S \subseteq R\left(A_{\phi, k, n}^{*}\right)^{\perp}$. Conversely, assume that $f \in R\left(A_{\phi, k, n}^{*}\right)^{\perp}$, then

$$
\left\langle\bar{\phi} \cdot\left(z_{1}^{k i_{1}} z_{2}^{k i_{2}} \ldots z_{n}^{k i_{n}}\right), f\right\rangle=\left\langle\left(z_{1}^{k i_{1}} z_{2}^{k i_{2}} \ldots z_{n}^{k i_{n}}\right), \phi \cdot f\right\rangle=0
$$


for each $\left(i_{1}, i_{2}, \ldots, i_{n}\right) \in \mathbb{Z}^{n}$. In view of the above expression, we obtain that $\phi \cdot f \in$ $\left[I-P_{k}\right]\left(L^{2}\left(\mathbb{T}^{n}\right)\right)$, where $P_{k}$ is the orthogonal projection of $L^{2}\left(\mathbb{T}^{n}\right)$ onto the closed subspace generated by $\left\{z_{1}^{k m_{1}} z_{2}^{k m_{2}} \ldots z_{n}^{k m_{n}}: m_{i} \in \mathbb{Z}, 1 \leq i \leq n\right\}$. The operator $\left(I-P_{k}\right)$ can be

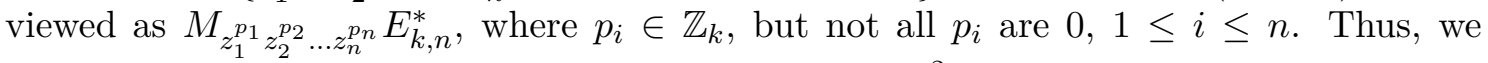

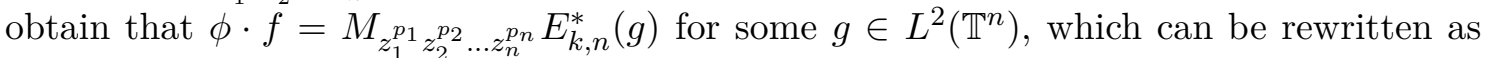
$f=\phi^{-1} \cdot\left(z_{1}^{p_{1}} z_{2}^{p_{2}} \ldots z_{n}^{p_{n}}\right) \cdot g\left(z_{1}^{k}, z_{2}^{k}, \ldots, z_{n}^{k}\right)$. This yields that $f \in S$. Hence, it follows that $S=R\left(A_{\phi, k, n}^{*}\right)^{\perp}$. This completes the proof.

Since the set $\left\{\phi \in L^{\infty}\left(\mathbb{T}^{n}\right):|\phi| \geq \epsilon>0\right.$ a.e. for some $\left.\epsilon\right\}$ is dense in $L^{\infty}\left(\mathbb{T}^{n}\right)$. Therefore, from the above lemma, one can conclude that $0 \in \sigma\left(A_{\phi, k, n}\right)$.

Lemma 3.22. The point spectra of $A_{\phi, k, n}$ and $A_{\phi\left(z_{1}^{k}, z_{2}^{k}, \ldots, z_{n}^{k}\right), k, n}$ coincide whenever $\phi \in$ $L^{\infty}\left(\mathbb{T}^{n}\right)$ is invertible.

Proof. Let $\lambda$ be an element of $\sigma_{p}\left(A_{\phi, k, n}\right)$. Then, $A_{\phi, k, n}(f)=\lambda f$, for some nonzero function $f \in L^{2}\left(\mathbb{T}^{n}\right)$. Under the assumption it is clear that $M_{\phi}(f) \neq 0$. Therefore, we can deduce that $M_{\phi} A_{\phi, k, n}(f)=\lambda M_{\phi} f$, which is equivalent to $A_{\phi\left(z_{1}^{k}, z_{2}^{k}, \ldots, z_{n}^{k}\right), k, n}(\phi \cdot f)=\lambda(\phi \cdot f)$. This implies that $\lambda \in \sigma_{p}\left(A_{\phi\left(z_{1}^{k}, z_{2}^{k}, \ldots, z_{n}^{k}\right), k, n}\right)$.

For the converse, assume that $\lambda \in \sigma_{p}\left(A_{\phi\left(z_{1}^{k}, z_{2}^{k}, \ldots, z_{n}^{k}\right), k, n}\right)$. Again, there exists a non zero function $g$ in $L^{2}\left(\mathbb{T}^{n}\right)$ such that $A_{\phi\left(z_{1}^{k}, z_{2}^{k}, \ldots, z_{n}^{k}\right), k, n}(g)=\lambda g$. Take $G=\phi^{-1} \cdot g$, clearly $G \neq 0$. Consequently, we get that $A_{\phi, k, n}(G)=\lambda(G)$. Hence, the desired result follows.

The next result gives a better visualization of spectrum of slant Toeplitz operators.

Theorem 3.23. Let $\phi$ be a function of the space $L^{\infty}\left(\mathbb{T}^{n}\right)$. Then, the spectrum of $A_{\phi, k, n}$ is the same as that of $A_{\phi\left(z_{1}^{k}, z_{2}^{k}, \ldots, z_{n}^{k}\right), k, n}$.

Proof. For the accomplishment of the proof, initially, we claim that $\sigma\left(A_{\phi, k, n}\right) \cup\{0\}=$ $\sigma\left(A_{\phi\left(z_{1}^{k}, z_{2}^{k}, \ldots, z_{n}^{k}\right), k, n}\right) \cup\{0\}$. In view of the property of the spectrum, we have

$$
\begin{aligned}
\sigma\left(A_{\phi, k, n}^{*}\right) \cup\{0\} & =\sigma\left(M_{\bar{\phi}} E_{k, n}^{*}\right) \cup\{0\}=\sigma\left(E_{k, n}^{*} M_{\bar{\phi}}\right) \cup\{0\} \\
& =\sigma\left(A_{\phi\left(z_{1}^{k}, z_{2}^{k}, \ldots, z_{n}^{k}\right), k, n}^{*}\right) \cup\{0\} .
\end{aligned}
$$

Thus, the above expression provides the desired claim. One can easily see that $0 \in$ $\sigma\left(A_{\phi\left(z_{1}^{k}, z_{2}^{k}, \ldots, z_{n}^{k}\right), k, n}\right)$. The preceding lemma, if $\phi$ is invertible, gives that $0 \in \sigma_{p}\left(A_{\phi, k, n}\right)$. If $\phi$ is not invertible, then $0 \in \sigma_{a p p}\left(A_{\phi, k, n}\right) \subseteq \sigma\left(A_{\phi, k, n}\right)$. Thus, the above observations bring out that $0 \in \sigma\left(A_{\phi, k, n}\right)$ for $\phi \in L^{\infty}\left(\mathbb{T}^{n}\right)$. This completes the proof.

Theorem 3.24. The spectrum of the $k^{\text {th }}$-order slant Toeplitz operator $A_{\phi, k, n}$, induced by an invertible function $\phi \in L^{\infty}\left(\mathbb{T}^{n}\right)$, contains a closed disc.

Proof. Let $\lambda$ be a nonzero element of $\mathbb{C}$ and the operator $\left(A_{\bar{\phi}^{-1}\left(z_{1}^{k}, \ldots, z_{n}^{k}\right), k, n}^{*}-\lambda I\right)$ be onto. Then, for $0 \neq g \in\left(I-P_{k}\right)\left(L^{2}\left(\mathbb{T}^{n}\right)\right)$, there exists a nonzero function $f \in L^{2}\left(\mathbb{T}^{n}\right)$ such that $\left(A_{\bar{\phi}^{-1}\left(z_{1}^{k}, \ldots, z_{n}^{k}\right), k, n}^{*}-\lambda I\right)(f)=g$, where $P_{k}$ is the projection of $L^{2}\left(\mathbb{T}^{n}\right)$ onto the closed subspace generated by the set $\left\{z_{1}^{k m_{1}} z_{2}^{k m_{2}} \ldots z_{n}^{k m_{n}}: m_{i} \in \mathbb{Z}, 1 \leq i \leq n\right\}$. The expression $\left(A_{\bar{\phi}^{-1}}^{*}\left(z_{1}^{k}, \ldots, z_{n}^{k}\right), k, n-\lambda I\right)(f)=g$ can be rewritten as

$$
\left(A_{\bar{\phi}^{-1}\left(z_{1}^{k}, \ldots, z_{n}^{k}\right), k, n}^{*}\right)(f)-\lambda P_{k}(f)-\lambda\left(I-P_{k}\right)(f)=g,
$$

equivalently,

$$
\left(M_{\phi^{-1}\left(z_{1}^{k}, \ldots, z_{n}^{k}\right)} E_{k, n}^{*}\right)(f)-\lambda P_{k}(f)-\lambda\left(I-P_{k}\right)(f)=g,
$$


The above expression along with the assumption yields that $\left(A_{\bar{\phi}^{-1}\left(z_{1}^{k}, \ldots, z_{n}^{k}\right), k, n}\right)(f)-$ $\lambda P_{k}(f) \in R\left(P_{k}\right) \cap R\left(I-P_{k}\right)=\{0\}$, where $R(T)$ represents the range set of $T$. Thus, the assumption, $0 \neq g \in\left(I-P_{k}\right)\left(L^{2}\left(\mathbb{T}^{n}\right)\right)$, helps to arrive at an inference that $\left(A_{\bar{\phi}^{-1}}^{*}\left(z_{1}^{k}, \ldots, z_{n}^{k}\right), k, n-\right.$ $\left.\lambda P_{k}\right)(f)=0$. Consequently, we get that

$$
\lambda E_{k, n}^{*} M_{\phi^{-1}}\left(\lambda^{-1}-M_{\phi} E_{k, n}\right)(f)=0 .
$$

Since, $M_{\phi}$ is invertible, $\lambda \neq 0$ and $E_{k, n}^{*}$ is an isometry, we get that

$\left(\lambda^{-1}-A_{\phi\left(z_{1}^{k}, z_{2}^{k}, \ldots, z_{n}^{k}\right), k, n}\right)(f)=0$, which implies that $\lambda^{-1} \in \sigma_{p}\left(A_{\phi\left(z_{1}^{k}, z_{2}^{k}, \ldots, z_{n}^{k}\right), k, n}\right)$.

If $\lambda \in \rho\left(A_{\bar{\phi}^{-1}\left(z_{1}^{k}, \ldots, z_{n}^{k}\right), k, n}^{*}\right)$, the resolvent of the operator $A_{\bar{\phi}^{-1}\left(z_{1}^{k}, \ldots, z_{n}^{k}\right), k, n}^{*}$, then, clearly, $\left(A_{\bar{\phi}^{-1}}^{*}\left(z_{1}^{k}, \ldots, z_{n}^{k}\right), k, n-\lambda I\right)$ is onto. Therefore, the above observation yields that

$$
D=\left\{\lambda^{-1}: \lambda \in \rho\left(A_{\bar{\phi}^{-1}\left(z_{1}^{k}, \ldots, z_{n}^{k}\right), k, n}^{*}\right)\right\} \subset \sigma_{p}\left(A_{\phi\left(z_{1}^{k}, z_{2}^{k}, \ldots, z_{n}^{k}\right), k, n}\right) .
$$

In view of the preceding Lemma 3.22, we have $D \subset \sigma_{p}\left(A_{\phi, k, n}\right)$. Since, the resolvent of an operator is an open subset of the complex plane, so $D$ is an open subset of $\mathbb{C}$ and contains an open disc. The compactness of the spectrum helps to point out that a closed disc is contained in the spectrum $\sigma\left(A_{\phi, k, n}\right)$ of $A_{\phi, k, n}$.

A trivial observation can be made from the proof of the above theorem in the following form.

Corollary 3.25. The spectrum of $A_{\phi, k, n}$ contains a closed disc of radius equals to $r\left(A_{E_{k, n}^{*}\left(\bar{\phi}^{-1}\right), k, n}\right)^{-1}$, where $r(\cdot)$ denotes the spectral radius.

For the particular choice $n=1$ and $k=2$, the results presented in the paper provide certain results proved by $\mathrm{Ho}[7,8]$ in the one variable case. At the end, we list the following illustrations related to the spectral radii and norms of these operators.

Example 3.26. For $\phi\left(z_{1}, z_{2}, \ldots, z_{n}\right)=\left(z_{1}^{k} z_{2}^{k} \ldots z_{n}^{k}+1\right)$, it can be seen that $|\phi|^{2}=2+$ $\left(z_{1}^{k} z_{2}^{k} \ldots z_{n}^{k}\right)+\overline{\left(z_{1}^{k} z_{2}^{k} \ldots z_{n}^{k}\right)}$, which yields that

$$
E_{k, n}\left(|\phi|^{2}\right)=2+\left(z_{1} z_{2} \ldots z_{n}\right)+\overline{\left(z_{1} z_{2} \ldots z_{n}\right)} .
$$

Similarly, we can compute $\Phi_{m}=2^{m-1}\left(2+\left(z_{1} z_{2} \ldots z_{n}\right)+\overline{\left(z_{1} z_{2} \ldots z_{n}\right)}\right)$. This implies that $\left\|\Phi_{m}\right\|_{\infty}=2^{m+1}$. Thus, the spectral radius $r\left(A_{\phi, k, n}\right)=\sqrt{2}$ and $\left\|A_{\phi, k, n}\right\|=2$. Therefore, the operator $A_{\phi, k, n}$ is not a normaloid.

Example 3.27. Let $\phi\left(z_{1}, z_{2}, \ldots, z_{n}\right)=\alpha+z_{1} z_{2} \ldots z_{n}, \alpha \in \mathbb{C}$. Then $\phi \in L^{\infty}\left(\mathbb{T}^{n}\right)$ and the spectral radius of $A_{\phi, k, n}$ is given by $r\left(A_{\phi, k, n}\right)=\sqrt{1+|\alpha|^{2}}$.

Acknowledgment. Insightful suggestions and the thorough review of the referee(s) are gratefully acknowledged. UGC, India grant with Ref. No. 1077/(CSIR-UGC, NET DEC. 2016) to the second author is also acknowledged.

\section{References}

[1] S.C. Arora and R. Batra, Generalized slant Toeplitz operators on $H^{2}$, Math. Nachr. 278 (4), 347-355, 2005.

[2] G. Datt and N. Ohri, Properties of slant Toeplitz operators on the torus, Malays. J. Math. Sci. 12, (2), 195-206, 2018.

[3] G. Datt and S.K. Pandey, Slant Toeplitz operators on Lebesgue space of $n$-dimensional Torus, Hokkaido Math. J. 49 (3), 363-389, 2020. 
[4] X. Ding, S. Sun and D. Zheng, Commuting Toeplitz operators on the bidisk, J. Funct. Anal. 263, 3333-3357, 2012.

[5] C. Gu and D. Zheng, The semi-commutator of Toeplitz operators on the bidisc, J. Operator Theory 38, 173-193, 1997.

[6] H. Guediri, Dual Toeplitz operators on the sphere, Acta Math. Sin. (Engl. Ser.) 19 (9), 1791-1808, 2013.

[7] M.C. Ho, Spectral properties of slant Toeplitz operators, Ph.D. thesis, PurdueUniversity, Indiana, 1996.

[8] M.C. Ho, Spectra of slant Toeplitz operators with continuous symbol, Michigan Math. J. 44, 157-166, 1997.

[9] Y.F. Lu and B. Zhang, Commuting Hankel and Toeplitz operators on the Hardy space of the bidisk, J. Math. Res. Exposition 30 (2), 205-216, 2010.

[10] V. Peller, Hankel operators and applications, Springer-Verlag, New York, 2003.

[11] W. Rudin, Function Theory in Polydisc, W.A. Benjamin Inc., New York-Amsterdam 1969.

[12] E.M. Stein and G. Weiss, Introduction to Fourier Analysis on Euclidean Spaces, Princeton Univ. Press, Princeton, NJ, 1971. 